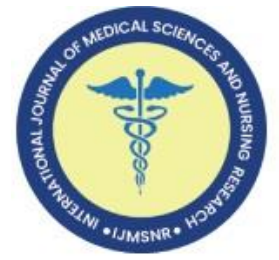

\title{
Hidden Markov Model of Evaluation of Break-Even Point of HIV patients: A Simulation Study
}

\author{
Mahalakshmi Rajendran $^{1}$, Senthamarai Kannan Kaliyaperumal ${ }^{2}$ (D) , Balasubramaniam Ramakrishnan ${ }^{3}$
}

${ }^{1,3}$ Research Scholar, Department of Statistics, Manonmaniam Sundaranar University, Abishekapatti, Tirunelveli, Tamil Nadu, India. ${ }^{2}$ Professor of Statistics, Department of Statistics, Manonmaniam Sundaranar University, Abishekapatti, Tirunelveli, Tamil Nadu, India.

\begin{abstract}
Background: The HIV virus carries projection of significant global population with specific estimations of the mathematical results of evolutionary methods which was presented in Tree Hidden Markov model (HMM).
\end{abstract}

Materials and Methods: Hidden Markov models used to model the progression of the disease among HIV infected people. The author predicts a Baum Welch Algorithm method through HMM that can assess an unknown state of transition.

Results: The Tree HMM model predicts the break down point starts once patient is infected with the HIV virus as it affects the immune system. The immune system drops more quickly in the initial inter arrival time when compared with the later time interval. The HIV virus length in the $\mathrm{n}^{\text {th }}$ state within regrouping is uncertain to occur in each state of the given model. A simulation study was done to assess the goodness of fit for the model.

Conclusion: The HIV virus length in the $\mathrm{n}^{\text {th }}$ state within regrouping is uncertain to occur in each state of the given model. The inter arrival censoring between each state is essential in each infected HIV patients. The outcome of this works states that health care expert can use this model for effective patient cares.

Keywords: expectation, hidden markov model, human immunodeficiency virus, immune system, transition

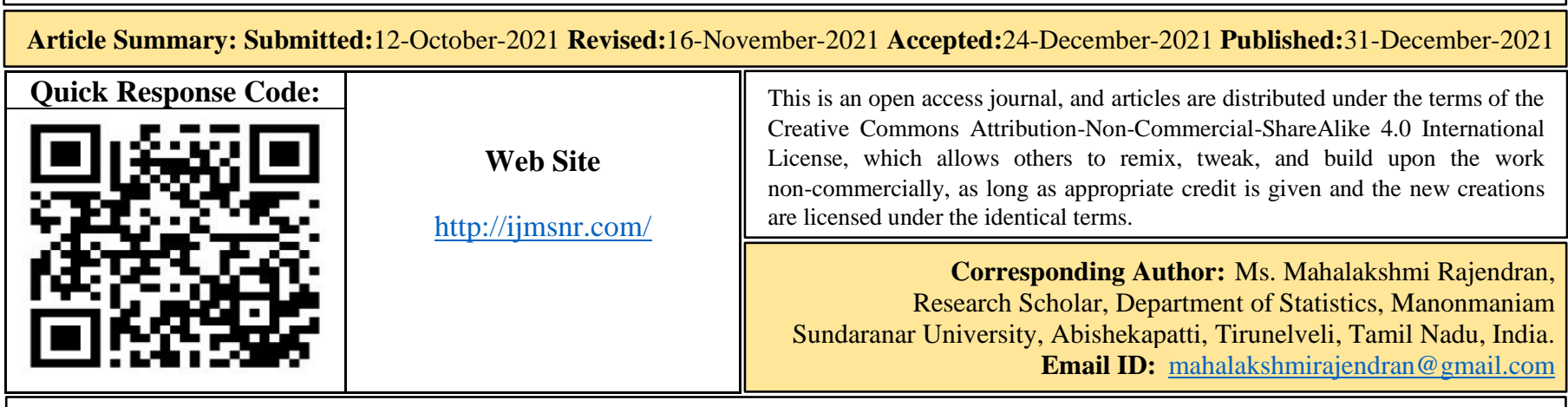

\section{Introduction}

Twenty-Six million people in 2020 June, were assessing the human immunodeficiency virus (HIV) antiretroviral therapy when compared to 2019 end an estimation of 25.4 million, an estimated $2.4 \%$ of increase was observed. Awareness among pregnant and breastfeeding women have been increased around $85 \%$ who have received ART living with HIV, this avoids HIV transmission to their newborns and also ensures their protective health. The $69^{\text {th }}$ World Wellbeing Gathering proposed a "Worldwide wellbeing area technique on HIV for 2016-2021". [1] The arrangement offered five vital headings, which are as per the following: data on designated activity of once pestilence and reaction, counteraction, treatment, and care, and exploration. The impact of mediations on the administrations required, guaranteeing uniformity for the populaces needing administrations, getting long haul subsidizing to pay the expenses of administrations, and speeding up the change to a manageable future are immensely significant contemplations. [2] UNAIDS has set a 2030 cutoff time for the destruction of the HIV pandemic, which will match with World Guides Day in 2014. As indicated by gauges, about 2.39 million individuals in India are tainted with HIV, making it the third most crowded country on the planet. South India was the main region to be hit by the HIV pandemic since it had the most noteworthy populace thickness at that point. [3]

Hidden Markov Model (HMM) is an extension of Markov model. Markov Model was named after Andrei Andreyevich Markov who lived in the year (1856-1922). Markov Chain is a statistical model where the data describes in sequence form. HMM is an especially embedded

How to cite this article: Rajendran M, Kaliyaperumal SK, Ramakrishnan B. Hidden Markov Model of Evaluation of Break-Even Point of HIV patients: A Simulation Study. Int J Med Sci and Nurs Res 2021;1(2):19-22 
under the umbrella of stochastic process where each state holds the Markov property. [4] The three main information to be observed in the HIV affected immune system is the parameter space, state space and state transition probability. [5]

Mathematical and Statistical models for infectious diseases commonly in the process of looking forward in estimating the epidemic which helps different public health sectors to plan optimally. Recent literature shows large number of literatures on Mathematical Models for communicable diseases. [6] A validated goodness of fit model (HMM) been used as an investigative to expect the diseases progression outcomes in infected cows. [7] Mathematical Modelling has been identified at the early stage of HIV epidemiological research, also concluded that theoretical research focuses on quantitative data on sequential changes in the mathematical distribution of sexual partner change along with other factors like variations in epidemiologic abundance in serum and emissions. [8] Mathematical Modelling suggests the cost effectiveness and time of HIV pandemic interventions, when given the right information to experimental trials. As the HIV pandemic is being a silent global threat since last four decades. [9] The HMM topology inference model denotes its graphical figures including the number of states with the association of symbols in each different state and state transitions with non-zero probabilities. Assuming the HMM model always specify the states prior to the information received. [10]

The Baum Welch Algorithm was published by Baum LE and along with coauthors who worked through his articles, even the name "Welch" appears as the coauthor that have been worked in developing this Baum Welch Algorithm. This algorithm was an example of Expectation Maximization (EM) algorithm. Mathematical methods associate to the algorithm along with an explanation as how the Baum Welch Algorithm fits the EM were also seen. [11 - 15]

We assume that the human immune system gets affected with HIV in a future state when the present state is already affected with HIV. The non-observable damage causing the immune system which leads to the HMM is the one to observe in this article. When the human system gets affected with HIV, it is represented by time $t=1$, which is the initial state of the process. At every time interval the human system moves from the current position to another position, i.e., $t=(1,2,3, \ldots \ldots)$, the transition probabilities are independent of the time $t$.

\section{Materials and Methods:}

Hidden Markov Model: [10] A continuous process to develop model parameters in the transition state to explain the respective time point in the infected patients. A Hidden Markov Model (HMM) is usually represented by $H M M: \mu=(A, B, \pi)$. This model tells us; the state transition probability, observational probability, probability of starting in a particular state. The Baum-Welch algorithm also known as EM-algorithm to emphasis on parameter estimation built on direct numerical maximum likelihood estimation. To maximize and find the posterior estimation of the hidden variables of HIV infected patients. The estimation depends on the assumption of the independent observations Tree HMM as seen in Figure-1. Transition variables defined as; $p t(i, j), 1 \leq t \leq T, 1 \leq i, j \leq N$
Figure-1 Hidden random variable shown with Tree HMM

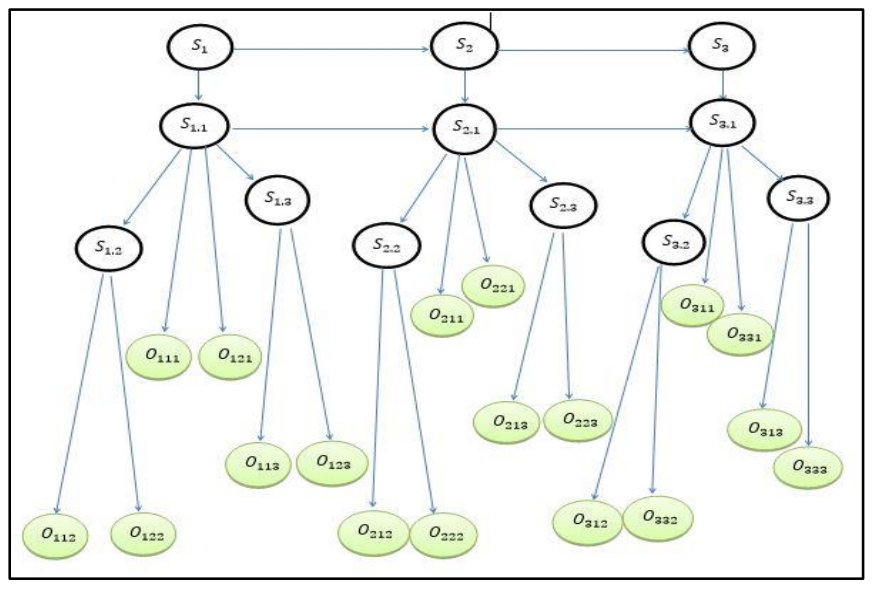

$a_{i j}^{\prime}=\frac{\text { Expected number of transitions from state } i \text { to } j}{\text { Expected number of transitions from state } i}$

$$
=\frac{\sum_{t=1}^{T} p_{t}(i, j)}{\sum_{t=1}^{T} \gamma_{i}(t)}
$$

$a_{i j}^{\prime}=\frac{\sum_{t=1}^{T} \alpha_{i}(t) a_{i j} b_{j}\left(O_{t+1}\right) \beta_{j}(t+1)}{\sum_{t=1}^{T} \alpha_{i}(t) \beta_{i}(t)}$

$\operatorname{Pr}(i j)=\operatorname{Pr}\left(S_{t=i}, S_{t+1=j} / O, \mu\right)$

$=\frac{\operatorname{Pr}\left(S_{t=i,} S_{t+1=j} / O, \mu\right)}{P(O / \mu)}$

$$
=\frac{\alpha_{i}(t) a_{i j} b_{i j o t} \beta_{j}(t+1)}{\sum_{m=1}^{N} \sum_{n=1}^{N} \alpha_{m}(t) a_{m n} b_{m n o t} \beta_{n}(t+1)}
$$

Equation (2) observes the probability of being at state $i$ at time $t$, and at state $j$ at time $t+1$, given the model $\mu$ and the observation $O$. Then, define $\gamma_{i}(t)$ this is the probability of being at state $i$ at time $t$, given the observation $O$ and the model $\mu$, as seen in equation (3),

$$
\begin{aligned}
\gamma_{i}(t) & =\operatorname{Pr}\left(\frac{S_{t=i}}{O}, \mu\right)=\sum_{j=1}^{N} \operatorname{Pr}\left(S_{t=i,} S_{t+1=j} / O, \mu\right) \\
& =\sum_{j=1}^{N} \operatorname{Pr}(i, j)
\end{aligned}
$$

The above equation (3) holds because $\gamma_{i}(t)$ is the expected number of transitions from state $i$ and $p t(i, j)$ is the expected number of transitions from $i$ to $j$. Given the above definitions we begin with an initial model $\mu$ and simply it for different states. 
$\pi_{i}^{\prime}=$ Probability of being at state $i$ at time $t=1 ;=\gamma_{i}(t)$

$a_{i j}^{\prime}=\frac{\text { Expected number of transitions from state } i \text { to } j}{\text { Expected number of transitions from state } i}$

$$
=\frac{\sum_{t=1}^{T} \operatorname{Pr}(i, j)}{\sum_{t=1}^{T} \gamma_{i}(t)}
$$

$b_{i j n}^{\prime}=\frac{\text { Expected number of transitions from } i \text { to } j \text { with } n \text { observed }}{\text { Expected number of transitions from } i \text { to } j}$

$$
=\frac{\sum_{t: O t=n, 1 \leq t \leq T} \operatorname{Pr}(i, j)}{\sum_{t=1}^{T} \operatorname{Pr}(i, j)}
$$

\section{Results and Discussion}

The three states are defined as; First state the initial state of HIV infection identified and under treatment (i.e., the person identified as HIV positive starting from the initial time period); Second State identified as the person infected under HIV after some period of initial time period; Third state observes the later time period of the infected person (i.e., the HIV infected persons are not aware of the diseases in them and identified it very lately). A simulation study was done to assess the goodness of fit for the model. The simulation was carried out using Mathcad Software and the graphical representation was figured through Minitab software.

Table-1 HIV infected patients risk observed in the three states as time increases

\begin{tabular}{|c|c|c|c|}
\hline $\begin{array}{c}\text { Time Per } \\
\text { Week }\end{array}$ & First State & $\begin{array}{c}\text { Second } \\
\text { State }\end{array}$ & $\begin{array}{c}\text { Third } \\
\text { State }\end{array}$ \\
\hline 1 & 2 & 3 & 4 \\
\hline 2 & 1.5 & 1.5 & 2 \\
\hline 3 & 1.33 & 1 & 1.333 \\
\hline 4 & 1.25 & 0.75 & 1 \\
\hline 5 & 1.2 & 0.6 & 0.8 \\
\hline 6 & 1.16 & 0.5 & 0.667 \\
\hline 7 & 1.14 & 0.429 & 0.571 \\
\hline 8 & 1.12 & 0.375 & 0.5 \\
\hline 9 & 1.11 & 0.333 & 0.444 \\
\hline 10 & 1.1 & 0.3 & 0.4 \\
\hline 20 & 1.05 & 0.15 & 0.2 \\
\hline 30 & 1.03 & 0.1 & 0.133 \\
\hline 40 & 1.02 & 0.075 & 0.1 \\
\hline 50 & 1.02 & 0.06 & 0.08 \\
\hline
\end{tabular}

The Tree HMM model predicts the break down point starts once patient is infected with the HIV virus as it affects the immune system. As the infected patient passes from one state to another the likelihood of high risk is more in the HIV patient as observed in Table-1 and Figure-2. The hidden nature of the virus is clearly observed in Table-1, stating the infected patient has a very less chance of survival as and when the time increases. The immune system drops more quickly in the initial inter arrival time when compared with the later time interval. The model finally concludes that, assessing the HIV patients at the initial time and state the likelihood of risk is less. As the time and state increases the likelihood of risk increases compared to the previous state.

\section{Figure-2 Three states of HIV infected patient's risk}

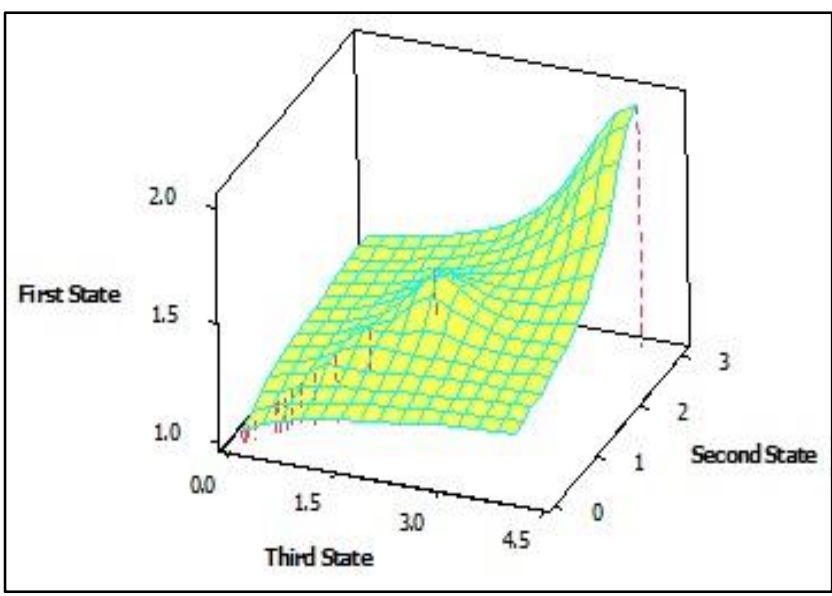

This simulation study attempts to make predictions of HIV patients and assess the performance of the model. For this, the dataset had taken from the World Health Organization Website. [2] The dataset had categorized into three subparts and renamed by states. The states are: S1 also known as the first state, is the initial state of HIV infection identified and under treatment. In this way S2, second state is the person infected under HIV; S3 is the state observes the later time period of the infected person.

Using the three states, the risk for the patients in the above states in every week was estimated and tabulated as shown in Table-1. The same estimated values were visualized using a three-dimensional graph as shown in Figure-2. Thus, the Hidden Markov Model was trained and the prediction was made using the Baum Welch Algorithm. [13, 14] The performance of the trained model was assessed. The risk of the patients in the three states also discussed.

\section{Conclusion}

The HIV virus carries projection of significant global population with specific estimations of the mathematical results of evolutionary methods which was presented in Tree HMM model. Our model assumes that the HIV infected patients are possibly of high risk in after state one. This HIV infected patients are of a single controlling strain in each state of the Tree HMM model. The HIV virus length in the $\mathrm{n}^{\text {th }}$ state within regrouping is uncertain to occur 
in each state of the given model. The inter arrival censoring between each state is essential in each infected HIV patients. The outcome of this works states that health care expert can use this model for effective patient cares.

Authors' contributions: MR, SKK, BR: Conception and Study design; MR: Acquisition of Data; MR, SKK, BR: Data processing, Analysis and Interpretation of Data; All authors MR, SKK, BR were drafting the article, revising it for intellectual content; All authors were checked and approved of the final version of the manuscript.

Here, MR - Mahalakshmi Rajendran; SKK - Senthamarai Kannan Kaliyaperumal, BR - Balasubramaniam Ramakrishnan

\section{Source of funding: None}

\section{Conflict of interest: None}

\section{References:}

1. AIDS and the sustainable development goals. Available from https://www.unaids.org/en/AIDS_SDGs [Accessed on: 20 August 2021]

2. A description about the disease HIV/AIDS. Available from: https://www.who.int/news-room/fact-sheets/detail/hiv-aids [Accessed on: 05 August 2021]

3. Rabiner LR and Juang BH. An introduction to hidden Markov models. IEEE ASSP Mag 1986;4-16. DOI: 10.1109/MASSP.1986.1165342

4. Sekar R, Amudhan M, Sivashankar M, Veeran R, Jayachandran C, Mythreyee M. Declining trend of HIV infection among the rural population of South India-A comparison of HIV prevalence in high risk and low risk groups. Journal of Infection 2011;63(1):9192. DOI: $10.1016 /$ j.jinf.2011.05.012

5. Norris JR. Markov Chains: Cambridge Series in Statistical and Probabilistic Mathematics, Series Number 2. Cambridge University Press 1998. ISBN-13: 978-0521633963

6. World Health Organization: HIV dataset Available from: https://www.who.int/data/gho/data/themes/hiv-aids [Accessed on: 28 August 2021]

7. Silva, CJ, Torres, DF. Modeling and optimal control of HIV/AIDS prevention through PrEP. Discrete Contin. Dyn. Syst. Ser 2017;11(1):119-141. DOI: 10.3934/dcdss.2018008

8. Ceres KM, Schukken YH, Gro"hn YT. Characterizing infectious disease progression through discrete states using hidden Markov models. PLoS ONE 2020;15(11):e0242683. PMID: 33216809

9. Abbas UL, Anderson RM, Mellors JW. Potential impact of antiretroviral therapy on HIV-1 transmission and AIDS mortality in resource-limited settings. J Acquir Immune Defic Syndr 2006;41(5):632-641. PMID: 16652038

10. Freedberg K, Possas C, Deeks S, Ross A, Rosettie K, Mascio M. The HIV cure research agenda: the role of mathematical modeling and cost effectiveness analysis. J Virus Erad 2015;1:245-249. PMID: 26878073
11. Rabiner LR. A tutorial on hidden Markov models and selected applications in speech recognition. Proceedings of the IEEE 1989;77(2):257-286. DOI: 10.1109/5.18626

12. Baum LE. An Inequality and Associated Maximization Technique in Statistical Estimation for Probabilistic Functions of Markov Processes. Inequalities and Maximization Technique 1972;3:1-8. Available from: https://files.library.northwestern.edu/public/Files/Baum.pdf

13. Baum LE, and Eagon JA. An Inequality with Applications to Statistical Estimation for Probabilistic Functions of Markov Processes and to a Model for Ecology. Bulletin of the American Mathematical Society 1967;73:360-363. DOI: 10.1090/S00029904-1967-11751-8

14. Baum LE, and Petrie T. Statistical Inference for Probabilistic Functions of Finite State Markov Chains. The Annals of Mathematical Statistics 1966;37:1554-1563. DOI: $10.1214 / \mathrm{aoms} / 1177699147$

15. Baum LE, and Sell GR. Growth Transformations for Functions on Manifolds. Pacific Journal of Mathematics 1968;27(2):211227. Available from: https://msp.org/pjm/1968/27-2/pjm-v27n2-p01-s.pdf

16. Baum, LE, Petrie T, Soules G and Weiss. A Maximization Technique Occurring in the Statistical Analysis of Probabilistic Functions of Markov Chains. The Annals of Mathematical Statistics 1970;41:164-171. DOI: $10.1214 /$ aoms/1177697196

Publish your research articles with

International Journal of Medical Sciences and Nursing Research

Website: http://ijmsnr.com/ 